\title{
GENERALIZED EXTERIOR ALGEBRAS
}

\section{N. MARCHUK}

PACS 98.62.Sb

(C) 2012
Steklov Mathematical Institute of the RAS

(8, Gubkin Str., Moscow 119991, Russia; e-mail: nmarchuk@mi. ras. ru; nmarchuk2005@ yandex)
Exterior algebras and differential forms are widely used in many fields of modern mathematics and theoretical physics. In this work, we define a notion of $N$-metric exterior algebra, which depends on $N$ matrices of structure constants. The usual exterior algebra (Grassmann algebra) can be considered as a 0-metric exterior algebra. The Clifford algebra can be considered as a 1-metric exterior algebra. $N$-metric exterior algebras for $N \geq 2$ can be considered as generalizations of the Grassmann and Clifford algebras. Specialists consider models of gravity that are based on a mathematical formalism with two metric tensors. We hope that the 2-metric exterior algebra considered in this work can be useful for the development of this model in gravitation theory and, especially, in the description of fermions in the presence of a gravity field.

\section{Clifford Algebras with a Nondiagonal Matrix of Structure Constants}

Let $\mathcal{E}$ be the $2^{n}$-dimensional vector space over a field $\mathbb{F}$ (of real or complex numbers) with basis elements

$$
\begin{aligned}
& e, e^{i}, e^{i_{1} i_{2}}, \ldots, e^{1 \ldots n} \\
& 1 \leq i \leq n, \quad 1 \leq i_{1}<i_{2} \leq n, \ldots
\end{aligned}
$$

enumerated by ordered multiindices of length from 0 to $n$. Let us also consider a real symmetric nondegenerate matrix $g \in \operatorname{Mat}(n, \mathbb{R})$ with elements $g^{i j}=g^{j i}$.

For elements of the vector space $\mathcal{E}$, we define the multiplication (Clifford multiplication) by the following rules:

1) $(\alpha U) V=U(\alpha V)=\alpha(U V)$ for $\forall U, V \in \mathcal{E}, \alpha \in \mathbb{F}$.

2) $(U+V) W=U W+V W, \quad W(U+V)=W U+W V$ for $\forall U, V, W \in \mathcal{E}$.

3) $(U V) W=U(V W)$ for $\forall U, V, W \in \mathcal{E}$.

4) $e U=U e=U$ for $\forall U \in \mathcal{E}$.

5) $e^{i} e^{j}+e^{j} e^{i}=2 g^{i j} e$ for $i, j=1, \ldots, n$.

6) $e^{i_{1}} \ldots e^{i_{k}}=e^{i_{1} \ldots i_{k}}$ for $1 \leq i_{1}<\cdots<i_{k} \leq n$.

Note that rules 1)-4) are the standard axioms of an associative algebra with identity element $e$.

Using these 6 rules, we can calculate products of any basis elements (1). In fact, to find the product $e^{i_{1}} \ldots e^{i_{k}}$ with unordered indices, we should rearrange factors in it using rule 5), taking into account that, from the product $e^{i} e^{j}$ in the case $i>j$ we get two summands: one that contains $e^{j} e^{i}$ and another one that contains $2 g^{i j} e$. As a result, the product $e^{i_{1}} \ldots e^{i_{k}}$ can be transformed with the aid of rules 1)-5) into a sum of products of elements $e^{i}$ with ordered indices

$e^{i_{1}} \ldots e^{i_{k}}=\alpha e^{l_{1}} \ldots e^{l_{p}}+\beta e^{m_{1}} \ldots e^{m_{q}}+\ldots$,

where $l_{1}<\cdots<l_{p}, m_{1}<\cdots<m_{q}, \ldots ; \alpha, \beta, \ldots$ are scalars. Now, in accordance with rule 6 ), we obtain

$e^{i_{1}} \ldots e^{i_{k}}=\alpha e^{l_{1} \ldots l_{p}}+\beta e^{m_{1} \ldots m_{q}}+\ldots$,

i.e., we get a linear combination of elements of basis (1) on the right-hand side. Finally, to calculate the product of any two elements of basis (1), we must write

$e^{i_{1} \ldots i_{k}} e^{j_{1} \ldots j_{r}}=e^{i_{1}} \ldots e^{i_{k}} e^{j_{1}} \ldots e^{j_{r}}$

and use the previous reasoning.

Example. Let us calculate the product

$$
\begin{aligned}
& e^{13} e^{234}=e^{1} e^{3} e^{2} e^{3} e^{4}=e^{1}\left(-e^{2} e^{3}+2 g^{23} e\right) e^{3} e^{4}= \\
& =-g^{33} e^{1} e^{2} e^{4}+2 g^{23} e^{1} e^{3} e^{4}=-g^{33} e^{124}+2 g^{23} e^{134} .
\end{aligned}
$$

Denote, by $E^{*}$, the vector space that spanned on the basis elements $e^{1}, \ldots, e^{n}$ with one index (we use notation $E^{*}$ because, in many applications, the space $E^{*}$ is the dual space to some initial pseudo-Euclidean space $E$ ). In the sequel, we see that the $2^{n}$ dimensional vector space $\mathcal{E}$ can be considered as the exterior algebra of the space $E^{*}$.

The vector space $\mathcal{E}$ over a field $\mathbb{F}$ with the defined operation of multiplication is called (real or complex, depending on $\mathbb{F}$ ) the Clifford algebra with the matrix of structure constants $g=\left\|g^{i j}\right\|$ and is denoted by $\mathcal{C}\left(E^{*}, g\right)$. The basis elements with one index $e^{1}, \ldots, e^{n}$ are called generators of the Clifford algebra $\mathcal{C} \ell\left(E^{*}, g\right)$, and the basis (1) is called the Clifford basis of the algebra $\mathcal{C}\left(E^{*}, g\right)$.

If the matrix $\left\|g^{i j}\right\|$ is diagonal, moreover with $r$ pieces of 1 and $q$ pieces of -1 on the diagonal, then the corresponding Clifford algebra is denoted by $\mathcal{C}(r, s)$ (by $\mathcal{C}(n)$ for $s=0$ ). 
One of the most important properties of Clifford algebras is the following: if an element $U \in \mathcal{C} \ell\left(E^{*}, g\right)$ is a linear combination of generators $U=u_{j} e^{j}$, then $U^{2}=\left(g^{i j} u_{i} u_{j}\right) e$, i.e. the square of this element is a scalar (proportional to the identity element $e$ ).

\section{An Exterior Multiplication of Elements of a Clifford Algebra $\mathcal{C}\left(E^{*}, g\right)$}

For elements of a Clifford algebra $\mathcal{C}\left(E^{*}, g\right)$, we define the operation of exterior multiplication, which is denoted by the symbol $\wedge$. For the products of generators, we put

$e^{i_{1}} \wedge e^{i_{2}} \wedge \ldots \wedge e^{i_{k}}=e^{\left[i_{1}\right.} e^{i_{2}} \ldots e^{\left.i_{k}\right]}$,

where the square brackets denote the operation of alternation of indices.

In particular, relation (2) yields the main identity of the Grassmann algebra

$e^{i} \wedge e^{j}=-e^{j} \wedge e^{i}$

\section{Example.}

$e^{i_{1}} \wedge e^{i_{2}}=\frac{1}{2}\left(e^{i_{1}} e^{i_{2}}-e^{i_{2}} e^{i_{1}}\right)=e^{i_{1}} e^{i_{2}}-g^{i_{1} i_{2}} e$.

It can be checked that formula (2) is equivalent to the formula

$e^{i_{1}} \wedge \ldots \wedge e^{i_{k}}=e^{i_{1}} \ldots e^{i_{k}}+\sum_{r=1}^{\left[\frac{k}{2}\right]} \frac{(-1)^{r}}{r !} Q^{r}\left(e^{i_{1}} \ldots e^{i_{k}}\right)$,

where

$Q\left(e^{i_{1}} \ldots e^{i_{k}}\right)=\sum_{1 \leq p<q \leq k}(-1)^{q-p-1} g^{i_{p} i_{q}} e^{i_{1}} \ldots$

$\ldots e^{\check{i}_{p}} \ldots e^{\check{i}_{q}} \ldots e^{i_{k}}$

The symbol ${ }^{-}$over the factor $e^{\check{i}_{p}}$ means that this factor in the product is omitted, $Q^{r}$ is the operation $Q$ applied $r$ times, and $\left[\frac{k}{2}\right]$ is the integer part of the number $k / 2$.

Formula (4) can be taken as the definition of exterior multiplication of Clifford algebra elements instead of formula (2).

Using formula (4), we may express Clifford products of generators $e^{i}$ in terms of exterior products of generators. Namely,

$e^{i_{1}} \ldots e^{i_{k}}=e^{i_{1}} \wedge \ldots \wedge e^{i_{k}}+\sum_{r=1}^{\left[\frac{k}{2}\right]} \frac{1}{r !} Q^{r}\left(e^{i_{1}} \wedge \ldots \wedge e^{i_{k}}\right)$, where

$Q\left(e^{i_{1}} \wedge \ldots \wedge e^{i_{k}}\right)=\sum_{1 \leq p<q \leq k}(-1)^{q-p-1} g^{i_{p} i_{q}} e^{i_{1}} \wedge \ldots$

$\ldots \wedge e^{\check{i}_{p}} \wedge \ldots \wedge e^{\check{i}_{q}} \wedge \ldots \wedge e^{i_{k}}$.

Example. From formula (6), in particular, we get

$e^{i_{1}} e^{i_{2}}=e^{i_{1}} \wedge e^{i_{2}}+g^{i_{1} i_{2}} e$,

$e^{i_{1}} e^{i_{2}} e^{i_{3}}=e^{i_{1}} \wedge e^{i_{2}} \wedge e^{i_{3}}+g^{i_{2} i_{3}} e^{i_{1}}-g^{i_{1} i_{3}} e^{i_{2}}+g^{i_{1} i_{2}} e^{i_{3}}$,

Formula (6) gives us a possibility to express elements of the Clifford basis (1) in terms of linear combinations of the following elements, which form a new basis of the Clifford algebra (Grassmann basis):

$e, e^{i}, e^{i_{1}} \wedge e^{i_{2}}, \ldots, e^{1} \wedge \ldots \wedge e^{n}$,

$1 \leq i \leq n, \quad 1 \leq i_{1}<i_{2} \leq n, \ldots$

Conversely, formula (4) gives us a possibility to express elements of the Grassmann basis (9) in terms of linear combinations of elements of the Clifford basis (1).

Now we can find the result of exterior multiplication

$e^{i_{1} \ldots i_{p}} \wedge e^{j_{1} \ldots j_{q}}$

of any elements of the Clifford basis. This can be done it three steps.

G1. Let us express basis elements $e^{i_{1} \ldots i_{p}}, e^{j_{1} \ldots j_{q}}$ in terms of elements of the Grassmann basis (9) and substitute the corresponding expressions into (10).

G2. Further, we calculate the exterior product of elements of the Grassmann basis. We get a result in the form of a sum of basis elements (9).

G3. Using formulas (4), we write down the result in terms of the Clifford basis (1).

\section{Example.}

$e^{13} \wedge e^{23}=\left(e^{1} \wedge e^{3}+g^{13} e\right) \wedge\left(e^{2} \wedge e^{3}+g^{23} e\right)=$

$=g^{23} e^{1} \wedge e^{3}+g^{13} e^{2} \wedge e^{3}+g^{13} g^{23} e=$

$=g^{23}\left(e^{13}-g^{13} e\right)+g^{13}\left(e^{23}-g^{23} e\right)+g^{13} g^{23} e=$

$=g^{23} e^{13}+g^{13} e^{23}-g^{13} g^{23} e$. 
On the same way, we may find the result of Clifford multiplication of any elements of the Grassmann basis

$$
\begin{aligned}
& \left(e^{i_{1}} \wedge \ldots \wedge e^{i_{p}}\right)\left(e^{j_{1}} \wedge \ldots \wedge e^{j_{q}}\right) \\
& i_{1}<\cdots<i_{p} ; j_{1}<\cdots<j_{q} .
\end{aligned}
$$

This also can be done in three steps.

C1. Let us express Grassmann basis elements $e^{i_{1}} \wedge \ldots \wedge$ $e^{i_{p}}, e^{j_{1}} \wedge \ldots \wedge e^{j_{q}}$ in terms of elements of the Clifford basis (1) using formulas (4) and substitute the corresponding expressions into (11).

C2. Further we calculate the Clifford product of elements of the Clifford basis. We get a result in the form of a sum of basis elements (1).

C3. Using formulas (6), we write down the result in terms of the Grassmann basis (9).

Example. It is easy to check that

$$
\begin{aligned}
& \left(e^{1} \wedge e^{3}\right)\left(e^{2} \wedge e^{3}\right)=-g^{33} e^{1} \wedge e^{2}+g^{23} e^{1} \wedge e^{3}- \\
& -g^{13} e^{2} \wedge e^{3}+\left(g^{13} g^{23}-g^{12} g^{33}\right) e .
\end{aligned}
$$

Therefore, we arrive at the $2^{n}$-dimensional vector space over a field $\mathbb{F}$ (real or complex numbers) with two operations of multiplication (Clifford multiplication and exterior multiplication) and with two bases (1) and (9). For both operations of multiplication, the associativity and distributivity axioms are satisfied. The basis element $e$ is the identity element for both operations.

If the matrix of structure constants $g=\left\|g^{i j}\right\|$ is diagonal, then each of formulas (4) and (6) gives us the relations

$e^{i_{1}} \ldots e^{i_{k}}=e^{i_{1}} \wedge \ldots \wedge e^{i_{k}} \quad i_{1}<\cdots<i_{k}$.

This means that bases (1) and (9) coincide. In this simple case, the described construction of a $2^{n}$-dimensional vector space with two operations of multiplication (exterior and Clifford) was considered by many authors (the first was H. Grassmann in 1877 [2]; see also [3]).

\section{Exterior Polymetric Algebras}

The Clifford algebra $\mathcal{C} \ell\left(E^{*}, g\right)$, considered with the only operation of exterior multiplication $\wedge$ and with the Grassmann basis (9), is an exterior algebra and denoted by $\Lambda\left(E^{*}\right)$. In previous section, we start from the Clifford algebra $\mathcal{C}\left(E^{*}, g\right)$ and arrive at the exterior algebra $\Lambda\left(E^{*}\right)$. In this section, we move in the opposite direction - from the exterior algebra $\Lambda\left(E^{*}\right)$ to the Clifford algebra $\mathcal{C}\left(E^{*}, g\right)$. On this way, we arrive at a new class of mathematical objects - exterior polymetric algebras.

- Let $E^{*}$ be an $n$-dimensional vector space with the basis $e^{1}, \ldots, e^{n}$, and let $\Lambda\left(E^{*}\right)$ be the exterior algebra of the vector space $E^{*}$ with the operation of exterior multiplication $\wedge: \Lambda\left(E^{*}\right) \times \Lambda\left(E^{*}\right) \rightarrow \Lambda\left(E^{*}\right)$ and with the Grassmann basis (9).

- The exterior multiplication satisfies the condition $e^{i} \wedge e^{j}=-e^{j} \wedge e^{i}$. The exterior algebra $\Lambda\left(E^{*}\right)$ is an associative algebra with identity element $e$. The dimension of the exterior algebra is equal to $2^{n}$.

Let us take a symmetric nondegenerate matrix $g=$ $\left\|g^{i j}\right\| \in \operatorname{Mat}(n, \mathbb{R})$. With the aid of this matrix and using formulas (6), we define new basis (1) of the algebra $\Lambda\left(E^{*}\right)$. This basis (Clifford basis) gives us a possibility to define Clifford multiplication for elements of the exterior algebra using rules 1)-6) (see page 422) and rules C1-C3 (see page 424 ).

So we again arrive at the algebra $\mathcal{C}\left(E^{*}, g\right)$ with two operations of multiplication - exterior and Clifford. However, the fact that now we start from the exterior algebra gives us a possibility to generalize the considered construction. Namely, we may consider algebras that have several matrices of structure constants $g_{1}, \ldots, g_{N}$.

In other words, let $E^{*}$ be an $n$-dimensional vector space, and let $\Lambda\left(E^{*}\right)$ be the exterior algebra of the space $E^{*}$ with operation of exterior multiplication (the dimension of the vector space $\Lambda\left(E^{*}\right)$ is equal to $\left.2^{n}\right)$ and with basis (9). Let also we have $N$ symmetric nondegenerate matrices $g_{1}, \ldots, g_{N} \in \operatorname{Mat}(n, \mathbb{R})$. We denote elements of these matrices by $g_{(k)}^{i j}, i, j=1, \ldots, n, k=1, \ldots, N$. With the aid of the matrices $g_{1}, \ldots, g_{N}$, we define $N$ operations of multiplication $\stackrel{k}{\vee}: \Lambda\left(E^{*}\right) \times \Lambda\left(E^{*}\right) \rightarrow \Lambda\left(E^{*}\right)$, $k=1, \ldots, N$. Every operation $\vee^{k}$ is an operation of the Clifford multiplication of elements of the exterior algebra that is defined with the aid of the matrix $g_{k}$ and with the use of rules 1)-6) (see page 422) and rules C1-C3 (see page 424 ).

The resulting unital associative algebra (with the exterior multiplication $\wedge$ and with Clifford multiplications $\left.\stackrel{1}{\vee}, \ldots, N^{N}\right)$ is called the exterior polymetric algebra or $N$ metric algebra and denoted by $\Lambda\left(E^{*}, g_{1}, \ldots, g_{N}\right)$. The 0 metric algebra coincide with the exterior algebra $\Lambda\left(E^{*}\right)$, and the 1-metric algebra coincide with the Clifford algebra $\mathcal{C}\left(E^{*}, g\right)$. The $N$-metric algebras $\Lambda\left(E^{*}, g_{1}, \ldots, g_{N}\right)$ for $N \geq 2$ can be considered as a generalization of the exterior algebra and the Clifford algebra. 
In the considered construction of the exterior polymetric algebra, the Grassmann basis (9) plays a primary role, and the Clifford bases play a secondary role.

A notion of the rank of an element of the exterior polymetric algebra corresponds to the Grassmann basis. Namely, an element $U \in \Lambda\left(E^{*}, g_{1}, \ldots, g_{N}\right)$ of the form

$U=\sum_{j_{1}<\ldots<j_{k}} u_{j_{1} \ldots j_{k}} e^{j_{1}} \wedge \ldots \wedge e^{j_{k}}$

is called an element of rank $k$. The set of elements of rank $k$ is the subset $\Lambda_{k}\left(E^{*}, g_{1}, \ldots, g_{N}\right)$ of the dimension $C_{n}^{k}$. We also define notions of even and odd elements of a polymetric algebra and

$$
\begin{aligned}
& \Lambda\left(E^{*}, g_{1}, \ldots, g_{N}\right)=\oplus_{k=0}^{n} \Lambda_{k}\left(E^{*}, g_{1}, \ldots, g_{N}\right)= \\
& =\Lambda_{\text {Even }}\left(E^{*}, g_{1}, \ldots, g_{N}\right) \oplus \Lambda_{\text {Odd }}\left(E^{*}, g_{1}, \ldots, g_{N}\right) .
\end{aligned}
$$

The dimensions of subspaces $\Lambda_{\text {Even }}\left(E^{*}, g_{1}, \ldots, g_{N}\right)$, $\Lambda_{\text {Odd }}\left(E^{*}, g_{1}, \ldots, g_{N}\right)$ are equal to $2^{n-1}$. The set of even elements $\Lambda_{\text {Even }}\left(E^{*}, g_{1}, \ldots, g_{N}\right)$ is the subalgebra of the exterior $N$-metric algebra.

The operations of conjugation of elements of a polymetric algebra also correspond to the Grassmann basis. In particular, the operation of pseudo-Hermitian conjugation $\ddagger: \Lambda\left(E^{*}, g_{1}, \ldots, g_{N}\right) \rightarrow \Lambda\left(E^{*}, g_{1}, \ldots, g_{N}\right)$ can be defined by the following rules:

- $\left(e^{a}\right)^{\ddagger}=e^{a}, a=1, \ldots, n ;$

- $(U \wedge V)^{\ddagger}=V^{\ddagger} \wedge U^{\ddagger}$;

- $(U+V)^{\ddagger}=U^{\ddagger}+V^{\ddagger}$;

- $(\lambda U)^{\ddagger}=\bar{\lambda} U^{\ddagger}$.

$U, V \in \Lambda\left(E^{*}, g_{1}, \ldots, g_{N}\right), \lambda \in \mathbb{F}$.

Consider some applications of the polymetric exterior algebra. In addition to Einstein's General Theory of Relativity, there are several alternative models of gravity. In particular, specialists consider models that are based on a mathematical formalism with two metric tensors. We hope that the 2-metric exterior algebra considered in this work can be useful for the development of these models in gravitation theory and, especially, for the description of fermions in the presence of a gravity field.

This work is partly supported by the grant NSh2928.2012.1 and by the Mathematical Division of the Russian Academy of Sciences (the program "Modern problems of theoretical mathematics").

1. N.G. Marchuk, Field Theory Equations and Clifford Algebras (R\&C Dynamics, Izevsk, 2009) (in Russian).

2. H. Grassmann, Math. Commun. 12, 375 (1877).

3. C. Doran, D. Hestenes, F. Sommen, and N. Van Acker, J. Math. Phys. 34, 3642 (1993).

4. I.M. Benn and R.W. Tucker, An Introduction to Spinors and Geometry with Applications to Physics (Hilger, Bristol, 1987).

5. P. Lounesto, Clifford Algebras and Spinors (Cambridge Univ. Press, Cambridge, 2001).

Received 15.07.11

\section{УЗАГАЛЬНЕНІ ЗОВНІШНІ АЛГЕБРИ}

\section{Н.Г. Марчук}

$\mathrm{P}$ е $з$ ю м е

У статті введено поняття поліметричної ( $N$-метричної) зовнішньої алгебри, яка залежить від $N$-матриць структурних констант. Звичайна зовнішня алгебра (алгебра Грассмана) розглядається як 0-метрична зовнішня алгебра. Алгебра Кліффорда розглядається як 1-метрична зовнішня алгебра. $N$-метричні зовнішні алгебри при $N>1$ розглядаються як узагальнення алгебри Грассмана і алгебри Кліффорда. Одним із підходів до теорії гравітації є модель, яка заснована на двох метричних тензорах. Автор сподівається, що запропонована в цій статті 2-метрична зовнішня алгебра буде корисною для розвитку вказаної моделі теорії гравітації та зокрема для опису ферміонів у присутності гравітаційного поля. 